\title{
Therapeutic Effects of Nigella Sativa Linn (Black Cumin) on Candida albicans Vaginitis
}

\author{
Farzaneh Adiban Fard ${ }^{1}$; Shahnaz Tork Zahrani ${ }^{2,}$; Alireza Akbarzadeh Bagheban ${ }^{3}$; Faraz \\ Mojab $^{4}$ \\ ${ }_{1}^{1}$ International Branch, University of Medical Sciences, Tehran, IR Iran \\ ${ }_{3}^{2}$ School of Nursing and Midwifery, Shahid Beheshti University of Medical Sciences, Tehran, IR Iran \\ ${ }^{3}$ School of Allied Medical Sciences, Shahid Beheshti University of Medical Sciences, Tehran, IR Iran \\ ${ }^{4}$ School of Pharmacy, Shahid Beheshti University of Medical Sciences, Tehran, IR Iran \\ *Corresponding author: Shahnaz Tork Zahrani, School of Nursing and Midwifery, Shahid Beheshti University of Medical Sciences, Tehran, IR Iran. Tel: + 98-2188202512, Fax: + 98- \\ 2188202521, E-mail:Zahrani@sbmu.ac.ir
}

Received: August 22, 2014; Revised: November 20, 2014; Accepted: November 25, 2014

\begin{abstract}
Background: Monilial Vaginitis is one of the most common gynecological problems among women all over the world causing 10 million consultations annually. Although the global prevalence of vaginitis is not clear, it has been reported that this infection becomes about twice more. The first cause of vaginitis in the Europe and the second cause in the United States are infections related to Candida albicans. Generally, the important cause of vaginitis is Candida albicans. Popular drugs regarding Candida albicans Vaginitis are topical ones such as Azoles. These drugs are known to cause severe adverse effects and high rate of relapse, in contrast some antifungal effects of Nigella Sativa L. without causing any adverse effect have been reported.

Objectives: The aim of this study was to determine the effect of Nigella Sativa Linn capsule in the treatment of Candida albicans vaginitis. Patients and Methods: This study was a randomized clinical trial, triple-blind, placebo controlled study performed on 100 women in Iran. Gynecological and microbiological evaluations were performed before and after the treatment. There were not statistically significant difference in signs and symptoms of monilial vaginitis before the treatment $(\mathrm{P}>0.05)$. Nigella Sativa Linn capsule and clotrimazole vaginal cream for the study group and placebo capsule and clotrimazole vaginal cream for the control group were prescribed for seven days. Data was obtained by interview and observation and analyzed with Chi-square, t-test, Mann-Whitney, Fisher-exact-test and McNemar tests. Results: There was a statistically significant difference in vaginal itching, discharge, irritation, vulvovaginal redness and inflammation after the treatment between the two groups and much more in the study group $(\mathrm{P}<0.001)$. However, the culture and wet mount (with $\mathrm{KOH}$ ) results showed much improvement in the study group after treatment.

Conclusions: The use of Nigella sativa L. capsule with clotrimazole vaginal cream was found more effective than clotrimazole vaginal cream alone, in most common symptoms and signs of this vaginitis. Therefore, Nigella Sativa L. capsule is suggested for the treatment of Candida albicans Vaginitis.
\end{abstract}

Keywords:Monilial Vaginitis; Nigella sativa; Clotrimazole

\section{Background}

Vaginitis is the most common gynecological problem among women seeking the treatment and causing 10 million physician visits annually (1). Although the global prevalence of vaginitis is not clear, it has been reported that this infection becomes about twice more (2). This infection is the first cause of vaginitis in Europe and the second cause in the United States (3). Candidiasis is the second common vaginal infection. Approximately $75 \%$ of all women experience it at least once (4) and $40-50 \%$ of women may experience twice in their life; whereas, $5 \%$ of them complain of recurrence of candidiasis vulvovaginitis (5). About 25 - $45 \%$ of patients have candida albicans vaginitis in Iran (6).

Currently, antifungal drugs are used for prevention and treatment of candidiasis that cause some drug-resistant species and encourage researchers to search for botani- cal drugs (7). Instead of Candida albicans infections, others are increasing that do not respond to treatment with common drugs such as clotrimazole and fluconazole (8). The vaginal cream clotrimazole $1 \%$ has some adverse effects including depression, painful urination, increase of hepatic enzymes, irritation and dermatitis. It has not been teratogen in animals, but was shown to be embryo toxic. Therefore, it is better to use with caution during pregnancy and breast-feeding (9). Herbal medicine has fewer adverse effects, less drug resistance, lower price and people tolerate them easier. A lot of medicinal plants are found in developing and developed countries (10).

Nigella Sativa Linn is a member of Ranunculaceae family, which grows in Asia and the Middle East. It has been used as food and natural drug (11). The ingredients of Nigella Sativa are Thymoquinone, Thymohydroquinone,

Copyright (c) 2015, Infectious Diseases and Tropical Medicine Research Center. This is an open-access article distributed under the terms of the Creative Commons Attribution-NonCommercial 4.0 International License (http://creativecommons.org/licenses/by-nc/4.0/) which permits copy and redistribute the material just in noncommercial usages, provided the original work is properly cited. 
Dithymoquinone and Thymol (12). The process of inflammation balances with lipo-oxygenizes (13) and Nigella's Thymoquinone inhibits the cycle of oxygenize $(14,15)$. Some pharmacological effects of Nigella Sativa L. include antimicrobial, anti-inflammatory, immune stimulation and anti-cancer properties (16).

\section{Objectives}

The aim of this study was to determine the effect of $\mathrm{Ni}$ gella Sativa Linn capsule in the treatment of Candida albicans vaginitis.

\section{Patients and Methods}

This study was a two-group study and triple-blind clinical trial blinded for investigator, drug producer and patients. The study protocol was approved with the ethical code (116/3705) and written consent was obtained from patients. The study was explained to patients, ensuring them to maintain their information and referring them if there were adverse effects or drug sensitivity. Patients did not know whether the capsules contained Nigella $S$. or placebo; vaginal cream clotrimazole was used for the both groups. Women were at reproductive age who complained for genital infections referring to Nader Clinic of Shahid Beheshti University of Medical Science in Tehran, Iran as a referral and governmental center from October 2013 to February 2014. Samples were chosen by convenience sampling and then allocated by stratified permutation blocks into the two groups of 44 women. By considering $10-15 \%$ potential loss of follow-up, 50 women in each group were allocated using the following Equation; Equation 1.

$$
n=\frac{\left(Z_{1-\frac{\alpha}{2}} \sqrt{2 \bar{P}(1-\bar{P})}+Z_{1+\beta} \sqrt{P_{1}\left(1-P_{1}\right)+P_{2}\left(1-P_{2}\right)}\right)^{2}}{\left(P_{1}-P_{2}\right)^{2}}
$$

$\mathrm{P}_{1}=0.90$

$\mathrm{P}_{2}=0.70$

$\alpha=0.05 \rightarrow Z_{\alpha}=1.96$

$\beta=0.2 \rightarrow \mathrm{Z}_{\beta}=0.84$

$\mathrm{n}_{1}=\mathrm{n}_{2}=44$

Expected Power $=80 \%$

Including: A and B (treatment with clotrimazole vaginal cream and Nigella S. capsule for the study group and vaginal cream clotrimazole and placebo capsule for the control group).

Inclusion criteria were:

- Women who aged 18 - 44 years.

- Having a symptom and sign of vulvovaginitis candidiasis and positive results for lab tests

- Not used antibiotics 14 days before the study

- Not used vaginal drugs 48 hours before the study

- Not used vaginal douche or quietuses 24 hours before the study

- Not used immunosuppressive drugs
- No trichomonal vaginitis, bacterial vaginitis or cervicitis and PID

- Not pregnant or breastfeeding

- Not having monilial vaginitis for four times or more than one year before the study

- Not premature menopause or mental retard

- Not having medical diseases such as diabetes, weakened immune system, thyroid disease and anemia

- Not using IUD and OCP

- Not using capsule two times or more

- Not menstruated and no abnormal uterine bleeding

The research was explained by one observer for patients who had all the inclusion and none of the exclusion criteria with positive results for candidiasis culture. After getting written consent from patients and filling checklists for signs, symptoms (content validity, Kappa 95\%) and demographic information, sample was taken in lithotomy position after sterilizing speculum from the vaginal fornix. Discharge specimen was put on two slides by swap. One slide was wet with a drop of $\mathrm{KOH}$ solution $10 \%$ for Whiff test and observing hypha of Candida albicans using microscope with $40 \times$ magnifications (calibrated). The second slide was wet with a drop of normal saline and put under microscope $(40 \times)$ to see clue cells of bacterial vaginosis and flagella of trichomonal vaginitis (Kappa 95\%). The other swap was put into normal saline tube for culture test by Chromagar (Merck, Germany), transferring to laboratory and preparing after 48 hours (Kappa 95\%). pH was determined by pH paper (Merck, Germany). Exclusion criteria were positive results for whiff test, not observing hypha and observing clue cell or flagella. The disease was proved with positive symptoms, signs and laboratory tests. Positive culture in Chromagar confirmed the diagnosis. For treatment, vaginal cream clotrimazole was prescribed seven nights in the both groups and Nigella capsule (500 $\mathrm{mg}$ ) was prescribed for the study group twice a day, seven nights and placebo capsule $(500 \mathrm{mg}$ ) was prescribed for the control group twice a day, seven nights. The second visit ( 2 - 7 days after the treatment) repeated as the first one, also patients were asked for any adverse effects. Successful treatment was considered with negative results for hypha slide and culture test. Statistical analysis was performed using SPSS software version 20.0 for windows (SPSS Inc., Chicago, USA). Quantitative data was presented with Mean \pm Standard Deviation (SD) and analyzed with McNemar, Chi-square, t-test, Fisher-exact-test and Mann-Whitney statistical tests. P value less than 0.05 was considered statistically significant.

\section{Results}

Age, weight and educational level were baseline variables. The populations mean age and weight were 30 - 31 years and 60 - $65 \mathrm{~kg}$ (Table 1 )

There was no significant difference $(P=0.66)$ between the two groups in educational level according to Chisquare test and most of them had diploma. Socioeco- 
nomic situation was an intervening variable measured by four variables including job (Chi-square, $\mathrm{P}=0.28$ ), housing-index (Chi-square, $\mathrm{P}=0.54$ ), income (Fisher exact test, $\mathrm{P}=0.15$ ) and number of family ( $\mathrm{t}$-test, $\mathrm{P}=0.80)$. There was no significant difference between the two groups.

There was no significant difference $(\mathrm{P}>0.05)$ between the two groups before and after the treatment in symptoms including vaginal itching, discharge, vulva irritation, dysuria and dyspareunia, according to Chi-square test. However, vaginal itching, vaginal discharge and vulva irritation were different between the two groups after treatment ( $\mathrm{P}$ $<0.001$ ) and decreased more in the study group. According to McNemar test, significant differences were observed in the both groups before and after the treatment $(\mathrm{P}<$ 0.05) and according to Chi-Square test, significant differences were observed between the groups in vaginal itching, discharge and vulva irritation $(\mathrm{P}<0.001)$. This showed that symptoms were improved after the treatment; however, improvement in the study group was more than the control group in three above-mentioned symptoms. The symptoms did not worsen in two groups (Table 2).

Table 1. Comparison of Mean Individual Characteristics in the Study and Control Groups

\begin{tabular}{lccccc}
\hline Groups & $\begin{array}{c}\text { Nigella Group } \\
\mathbf{n}=\mathbf{5 0}\end{array}$ & $\begin{array}{c}\text { Placebo Group } \\
\mathbf{n}=\mathbf{5 0}\end{array}$ & \multicolumn{3}{c}{$\begin{array}{c}\text { Independent } \\
\text { t-test }\end{array}$} \\
\cline { 2 - 6 } & Mean $\pm \mathbf{S D}$ & Mean $\pm \mathbf{S D}$ & $\mathbf{t}$ & $\mathbf{D f}$ & $\mathbf{P}$ \\
\hline Age & $30.82 \pm 6.58$ & $30.66 \pm 6.61$ & 0.12 & 98 & 0.90 \\
Weight & $63.34 \pm 12.86$ & $63.26 \pm 10.93$ & 0.03 & 98 & 0.97 \\
Height & $160.44 \pm 4.70$ & $160.42 \pm 4.48$ & 0.02 & 98 & 0.98 \\
\hline
\end{tabular}

Table 2. Comparison of Distribution of Symptoms in the Study and Control Groups ${ }^{\text {a }}$

\begin{tabular}{lcccc}
\hline $\begin{array}{l}\text { Group Symp- } \\
\text { toms (before) }\end{array}$ & $\begin{array}{c}\text { Nigella Sativa Group } \\
\text { (After) }\end{array}$ & \multicolumn{2}{c}{$\begin{array}{c}\text { Placebo Group } \\
\text { (After) }\end{array}$} \\
\cline { 2 - 5 } Itching & + & - & + & - \\
+ & $2(4)$ & $44(88)$ & $13(26)$ & $32(64)$ \\
- & $0(0)$ & $4(8)$ & $1(2)$ & $4(8)$ \\
Discharge & & & & \\
+ & $1(2)$ & $47(94)$ & $18(36)$ & $27(54)$ \\
\hline- & $0(0)$ & $2(4)$ & $1(2)$ & $4(8)$ \\
Irritation & & & & \\
+ & $1(2)$ & $28(56)$ & $13(26)$ & $5(10)$ \\
\hline- & $0(0)$ & $21(42)$ & $7(14)$ & $25(50)$ \\
\hline Dysuria & & & & \\
+ & $4(8)$ & $7(14)$ & $7(14)$ & $4(8)$ \\
\hline- & $0(0)$ & $39(78)$ & $1(2)$ & $38(76)$ \\
\hline Dyspareunia & & & & \\
+ & $8(16)$ & $8(16)$ & $6(12)$ & $4(8)$ \\
\hline & $0(0)$ & $34(68)$ & $0(0)$ & $40(80)$ \\
\hline a All Values are Presented as No. (\%). & &
\end{tabular}

Inflammation and redness in vulve, pustular papular wound in vulve, abnormal cervix, inflammation and redness in grain and pustular papular wound in grain had no significant differences between the two groups ( $\mathrm{P}>$ 0.05 ) before and after the treatment. However, inflammation and redness in vulve was different in the two groups after the treatment $(\mathrm{P}<0.001)$ and decreased more in the study group. According to McNemar test, significant differences were observed in the both groups before and after the treatment $(\mathrm{P}<0.05)$ and signs were improved after the treatment. According to Chi square test, significant differences were observed between two groups regarding inflammation and redness in vulve $(\mathrm{P}=0.002)$. The signs did not worsen in the two groups (Table 3 ).

Table 3. Comparison of Distribution of Signs in the Study and Control Groups ${ }^{\mathrm{a}}$

\begin{tabular}{|c|c|c|c|c|}
\hline \multirow[t]{2}{*}{ Group Signs (before) } & \multicolumn{2}{|c|}{$\begin{array}{l}\text { Nigella Sativa } \\
\text { Group (After) }\end{array}$} & \multicolumn{2}{|c|}{$\begin{array}{c}\text { Placebo } \\
\text { Group (Af- } \\
\text { ter) } \\
\end{array}$} \\
\hline & + & - & + & - \\
\hline \multicolumn{5}{|c|}{$\begin{array}{l}\text { Inflammation and Redness } \\
\text { in vulve }\end{array}$} \\
\hline+ & $1(2)$ & $44(88)$ & $11(22)$ & $38(76)$ \\
\hline- & $0(0)$ & $5(10)$ & $0(0)$ & $1(2)$ \\
\hline \multicolumn{5}{|c|}{$\begin{array}{l}\text { pustular papular wound in } \\
\text { vulve }\end{array}$} \\
\hline+ & $0(0)$ & $15(30)$ & $1(2)$ & $17(34)$ \\
\hline- & $0(0)$ & $35(70)$ & $0(0)$ & $32(64)$ \\
\hline \multicolumn{5}{|l|}{ Abnormal cervix } \\
\hline+ & $0(0)$ & $9(18)$ & $0(0)$ & $6(12)$ \\
\hline- & $0(0)$ & $41(82)$ & $0(0)$ & $44(88)$ \\
\hline \multicolumn{5}{|c|}{$\begin{array}{l}\text { Inflammation and redness } \\
\text { in grain }\end{array}$} \\
\hline+ & $0(0)$ & $1(2)$ & $1(2)$ & $4(8)$ \\
\hline- & $0(0)$ & $49(98)$ & $0(0)$ & $45(90)$ \\
\hline \multicolumn{5}{|c|}{$\begin{array}{l}\text { pustular papular wound in } \\
\text { grain }\end{array}$} \\
\hline+ & $0(0)$ & $0(0)$ & $0(0)$ & $1(2)$ \\
\hline - & $0(0)$ & $50(100)$ & $0(0)$ & $49(98)$ \\
\hline
\end{tabular}

Wet mount with $\mathrm{KOH}$, culture test with Chromagar, wet mount with normal saline and $\mathrm{pH}$ test had no significant differences $(\mathrm{P}>0.05)$ between the two groups before the treatment. Wet mount of patients with normal saline had always negative results before and after the treatment, so there was no statistical analysis. $\mathrm{pH}$ of patient's discharge was always positive and natural before and after the treatment, so there was no statistical analysis. According to McNemar test and Chi-Square, significant differences were observed between the both groups before and after the treatment for wet mount with $\mathrm{KOH}$ and Chromagar culture $(\mathrm{P}<0.001)$; wet mount with saline and Chro- 
magar culture improved after the treatment in the study group more than the placebo group (Table 4).

Table 4. Comparison of Distribution of lab Tests in the Study and Control Groups ${ }^{a}$

\begin{tabular}{lcccc}
\hline Groups Lab tests & \multicolumn{2}{c}{ Nigella S. Group } & \multicolumn{2}{c}{ Placebo Group } \\
\cline { 2 - 5 } & Before & After & Before & After \\
\hline Wet mount (KOH) & & & & \\
+ & $50(100)$ & $1(2)$ & $50(100)$ & $25(50)$ \\
\hline- & $0(0)$ & $49(98)$ & $0(0)$ & $25(50)$ \\
Chromagar Culture & & & & \\
+ & $50(100)$ & $1(2)$ & $50(100)$ & $19(38)$ \\
\hline & $0(0)$ & $49(98)$ & $0(0)$ & $31(62)$ \\
\hline a & & & &
\end{tabular}

\section{Discussion}

The rising prevalence of candida albicans antibiotic resistance increased the need for new and safe drugs for the treatment of vulvovaginitis candidiasis. However there are some in vitro and in vivo researches on Nigella Sativa Linn and its medicinal effects, there is no study about the effect of this herb on candida albicans vaginitis in human.

The results of demographic variables are in agreement with the present study. Wallimg's study (17) in the United States showed that most candidiasis vaginitis samples were not pregnant, which does not agree with the present study. The difference may be because of pregnancy age in Iran, which is lower than Western countries. According to education level of patients that was diploma, Simbar et al. (18) in their study obtained the mean education year of test group as $9.05 \pm 4.37$ and for control group as 9.52 \pm 3.61 , which was lower than diploma; the difference may be due to improved education level.

In the current study, all symptoms decreased in the two groups and both treatments improved the symptoms, but more improved in the study group, compared to the placebo group in symptoms including vaginal itching and vaginal discharge and equal effect on other symptoms in both group was observed. The result showed that improvement of all signs was the same in the two groups and both treatments improved the symptoms, but more in the study group compared to placebo group in the inflammation and redness in vulve and a same result achieved in other signs. Comparison of differential mean in number of symptoms and signs after the treatment showed that improving in the symptoms of vulvovaginal candidiasis in Nigella group was a decrease of 2.68 units $(\mathrm{P}<0.001)$, however, it was 1.24 units in the placebo group, which means that Nigella could improve the symptoms two times more than placebo. According to McNemar test, significant differences were observed in the both groups before and after the treatment for wet mount with $\mathrm{KOH}$ and Chromagar culture $(\mathrm{P}<0.001)$, however, Chi-Square test showed significant differences between the two groups after the treatment $(\mathrm{P}<0.001)$. Wet mount with $\mathrm{KOH}$ and Chromagar culture improved after the treatment in Nigella group more than the placebo group. The result of this article emphasized the Nigella's mechanism. Although there is no research about the therapeutic effect of Nigella Sativa L. on Candida albicans vaginitis in humans, some herbal drug such as honey, cinnamon and Myrtus were more effective than clotrimazole.

This study can produce scientific evidence for using in traditional medicine; also, can be a base for further studies to develop effective anti-fungal drugs (strong points). We could not prepare oil capsules of Nigella Sativa (weak point). Finally, regarding the findings of this study, this drug can be used with confidence for treatment of candidiasis vaginitis in patients who tend to treatment with herbal medications or cases resistance to clotrimazole.

\section{Acknowledgements}

The authors of this study would like to thank staffs of Nader clinic. Authors also thank Dr. Nayebali Ahmadi for his cooperation in teaching microscopic analysis.

\section{Author's Contributions}

Farzaneh Adiban Fard designed the study and wrote the manuscript. Also, she examined the patients and performed the lab tests. Shahnaz Tork Zahrani revised the manuscript.

\section{Funding/Support}

This study was supported by the International Branch, Shahid Beheshti University of Medical Sciences, Tehran, Iran.

\section{References}

1. Ryanm KJ, Kistner RW. Kistner's Gynecology and Women's Health. 7 ed: Mosby; 1999.

2. Sobel JD. Vulvovaginal candidosis. Lancet. 2007;369(9577):1961-71.

3. Babic M, Hukic M. Candida albicans and non-albicans species as etiological agent of vaginitis in pregnant and non-pregnant women. Bosn J Basic Med Sci. 2010;10(1):89-97.

4. Berek JS. Berek and Novak's gynecology.14 edUnited States: Wolters Kluwer/Lippincott Williams Wilkins; 2012.

5. Ahmad A, Khan AU. Prevalence of Candida species and potential risk factors for vulvovaginal candidiasis in Aligarh, India. Eur J Obstet Gynecol Reprod Biol. 2009;144(1):68-71.

6. Akbarzadeh M, Bonyadpoure B, Pacshir K, Mohagheghzadeh A. Causes and clinical symptoms of vaginal candidiasis in patients referring to selective clinics of Shiraz University of Medical Sciences (2009). Arak Med Uni J. 2010;13(3):12-20.

7. Nodoushan AJ, Dehghani M, Mirbagheri SM. In vitro antifungal effect of aqueous Garlic (Allium sativum) extract and its combination with fluconazole against five common clinical Candida isolated from candidiasis lesions. J Kerman Uni Med Sci. 2007;14(3):153-62.

8. Gibbs RS, Danforth DN, Karlan B, Haney AF. Danforth's obstetrics and gynecology.Philadelphia: Lippincott Williams and Wilkins,; 2009

9. Lowinson JH, Ruiz P. A comprehensive textbook of drug information 4 ed. Iran pharma editor. Tehran: Teimourzadeh Tabib; 2004. 


\section{Adiban Fard F et al.}

10. Vermani K, Garg S. Herbal medicines for sexually transmitted diseases and AIDS.J Ethnopharmacol. 2002;80(1):49-66.

11. Salem ML. Immunomodulatory and therapeutic properties of the Nigella sativa L. seed. Int Immunopharmacol. 2005;5(1314):1749-70.

12. Fallah Huseini H, Amini M, Mohtashami R, Ghamarchehre ME, Sadeqhi Z, Kianbakht S, et al. Blood pressure lowering effect of Nigella sativa L. seed oil in healthy volunteers: a randomized, double-blind, placebo-controlled clinical trial. Phytother Res. 2013;27(12):1849-53.

13. Williams CS, Mann M, DuBois RN. The role of cyclooxygenases in inflammation, cancer, and development. Oncogene. 1999;18(55):7908-16.

14. Mansour M, Tornhamre S. Inhibition of 5-lipoxygenase and leukotriene $C 4$ synthase in human blood cells by thymoquinone. $J$ Enzyme Inhib Med Chem. 2004;19(5):431-6.

15. Houghton PJ, Zarka R, de las Heras B, Hoult JR. Fixed oil of Nigella sativa and derived thymoquinone inhibit eicosanoid generation in leukocytes and membrane lipid peroxidation. Planta Med. 1995;61(1):33-6.
16. Salem EM, Yar T, Bamosa AO, Al-Quorain A, Yasawy MI, Alsulaiman RM, et al. Comparative study of Nigella Sativa and triple therapy in eradication of Helicobacter Pylori in patients with non-ulcer dyspepsia. Saudi J Gastroenterol.2010;16(3):207-14.

17. Wallimg AD. A cost effective strategy for diagnosing vaginal candidiass. Am Fam Physician. 1999;5:111-2.

18. Simbar M, Azarbad Z, Mojab F, Majd HA. A comparative study of the therapeutic effects of the Zataria multiflora vaginal cream and metronidazole vaginal gel on bacterial vaginosis. Phytomedicine. 2008;15(12):1025-31. 